\title{
Perioperative Transesophageal Echocardiographic Diagnosis of Membranous Interventricular Septal Aneurysm in Two Patients
}

\author{
${ }^{1}$ Vikas Dutta, ${ }^{2}$ Sunder L Negi, ${ }^{3}$ Goverdhan Dutt Puri, ${ }^{4}$ Rana S Singh
}

\begin{abstract}
Aneurysms of interventricular septum are a rare anomaly usually seen as an incidental finding on echocardiography. Rarely, they can cause right ventricular outflow tract obstruction. They can present in patients having other cardiac lesions. Diagnosis of interventricular septal aneurysm is not straightforward. They can be confused with sinus of valsalva aneurysm or ventricular septal defect on transthoracic echocardiography. Transesophageal echocardiography (TEE) provides a better alternative to delineate the interventricular septal aneurysm. We describe the TEE diagnosis of membranous interventricular septal aneurysm in two dissimilar patients who presented for cardiac surgery at our cardiac center.
\end{abstract}

Keywords: Interventricular septum, Septal aneurysm, Transesophageal echocardiography.

How to cite this article: Dutta V, Negi SL, Puri GD, Singh RS. Perioperative Transesophageal Echocardiographic Diagnosis of Membranous Interventricular Septal Aneurysm in Two Patients. J Perioper Echocardiogr 2016;4(1):14-16.

Source of support: Nil

Conflict of interest: None

\section{INTRODUCTION}

Ventricular septum aneurysms have been described to occur in membranous and muscular part of interventricular septum. Aneurysm of membranous part is a congenital anomaly, whereas most of the aneurysms of the muscular septum are seen after myocardial infarction. ${ }^{1}$ Angiography was the only method of diagnosis before the introduction of echocardiography. ${ }^{2}$ Two-dimensional (2D) echocardiography can detect the aneurysms of ventricular septum with a sensitivity of $70 \%{ }^{3}$ We report

\footnotetext{
1,2Senior Resident, ${ }^{3,4}$ Professor

1-3Department of Anaesthesiology and Intensive Care Postgraduate Institute of Medical Education and Research Chandigarh, India

${ }^{4}$ Department of Cardiovascular and Thoracic Surgery Postgraduate Institute of Medical Education and Research Chandigarh, India

Corresponding Author: Goverdhan Dutt Puri, Professor Department of Anaesthesiology and Intensive Care Postgraduate Institute of Medical Education and Research Chandigarh, India, Phone: +919915647637, e-mail: gdpuri007@ hotmail.com
}

the transesophageal echocardiography (TEE) findings of membranous interventricular septal aneurysm seen in two dissimilar patients.

\section{CASE REPORTS}

\section{Case 1}

A 23-year-old male patient was admitted with a history of dyspnea on exertion. Chest X-ray showed cardiomegaly. On auscultation, there was a $3 / 6$ holodiastolic murmur in the third left intercostal space. Diagnosis of bicuspid aortic valve with severe aortic regurgitation with sinus of valsalva aneurysm was made on transthoracic echocardiography (TTE). The patient was posted for aortic valve replacement with repair of sinus of valsalva aneurysm. Transesophageal echocardiography was done after induction of anesthesia. Aortic valve short-axis view showed a bicuspid aortic valve and a membranous structure prolapsing into right ventricle (RV) during systole (Fig. 1A). Color Doppler interrogation showed no communication between RV and membranous structure (Fig. 1B). Midesophageal long-axis view showed communication between left ventricular outflow tract (LVOT) and aneurysm (Fig. 2). The aneurysm was not communicating with $\mathrm{RV}$, and there was no dilatation of sinus of valsalva. Thus a diagnosis of aneurysm of interventricular septum was made. The patient underwent aortic valve replacement and resection of aneurysm with primary closure of defect. The patient had uneventful postoperative course.

\section{Case 2}

A 2-year-old male child was admitted with history of recurrent chest infections. Chest X-ray was normal. On auscultation, there was a $3 / 6$ systolic ejection murmur in second left intercostal space with widely split S2. Transthoracic echocardiography showed $4 \mathrm{~mm}$ perimembranous ventricular septal defect (VSD) with valvular pulmonary stenosis. Mean gradient across pulmonary valve was $43 \mathrm{~mm} \mathrm{Hg}$. After induction of anesthesia TEE was done. Midesophageal five-chamber view showed a defect in interventricular septum and a membranous structure bulging into RV during systole (Fig. 3A). Midesophageal RV inflow outflow view showed aneurysm of interventricular septum bulging 

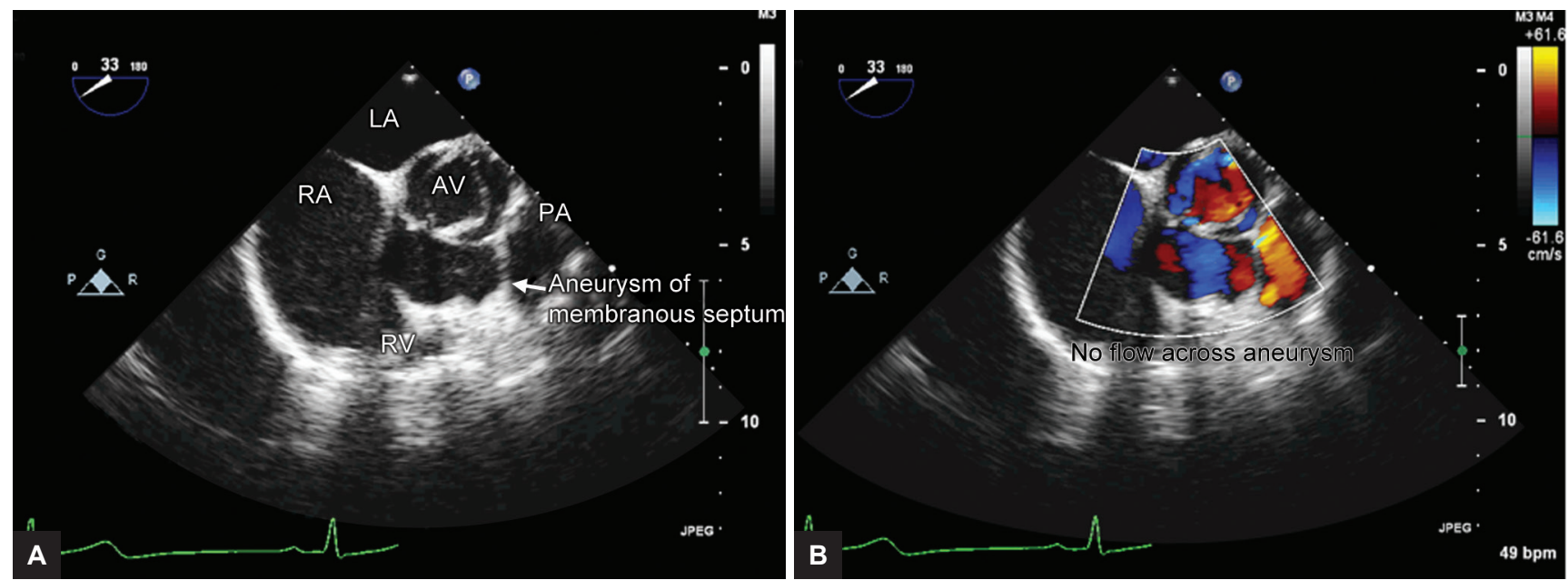

Figs 1A and B: (A) Aortic valve short-axis view showing bicuspid aortic valve and membranous septal aneurysm;

(B) no flow across the aneurysm

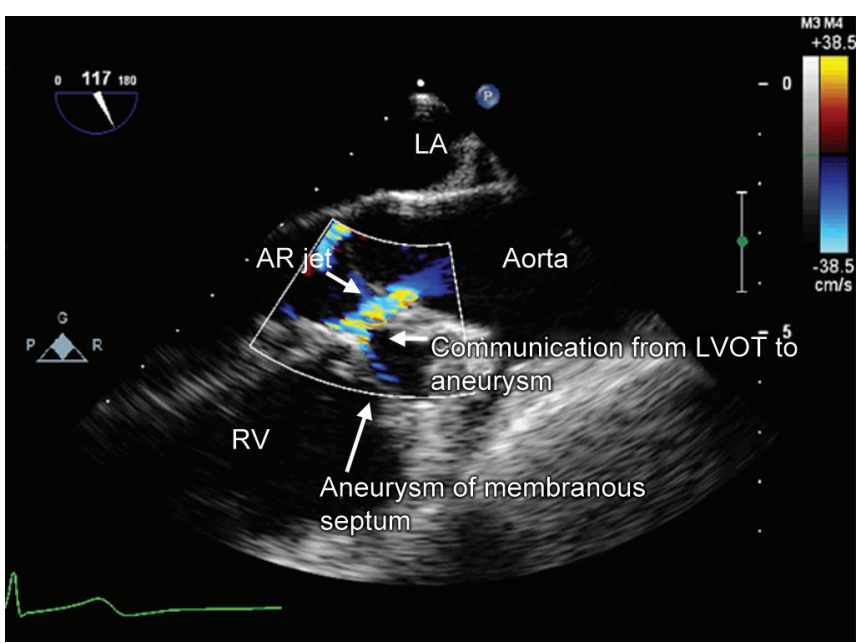

Fig. 2: Midesophageal long-axis view showing communication between left ventricular outflow tract and membranous septal aneurysm with normal sinus of valsalva

into RV during systole (Fig. 3B). Transesophageal echocardiography also revealed an ostium secundum atrial septal defect (ASD) with left-to-right shunt and left main coronary arising from the noncoronary cusp (Fig. 4). Aneurysm resection, VSD closure (created after aneurysm resection), ASD closure, and pulmonary valvotomy were done. The patient was discharged from the hospital uneventfully.

\section{DISCUSSION}

Aneurysm of the membranous interventricular septum is a rare anomaly and is usually benign. It can potentiate tricuspid regurgitation, aortic valve prolapse, and right ventricular outflow tract (RVOT) obstruction. ${ }^{4}$ The natural history of aneurysm of membranous ventricular septum is not well understood. Spontaneous closure of perimembranous VSD can result in aneurysm formation with or without residual defect. ${ }^{5}$ In the first
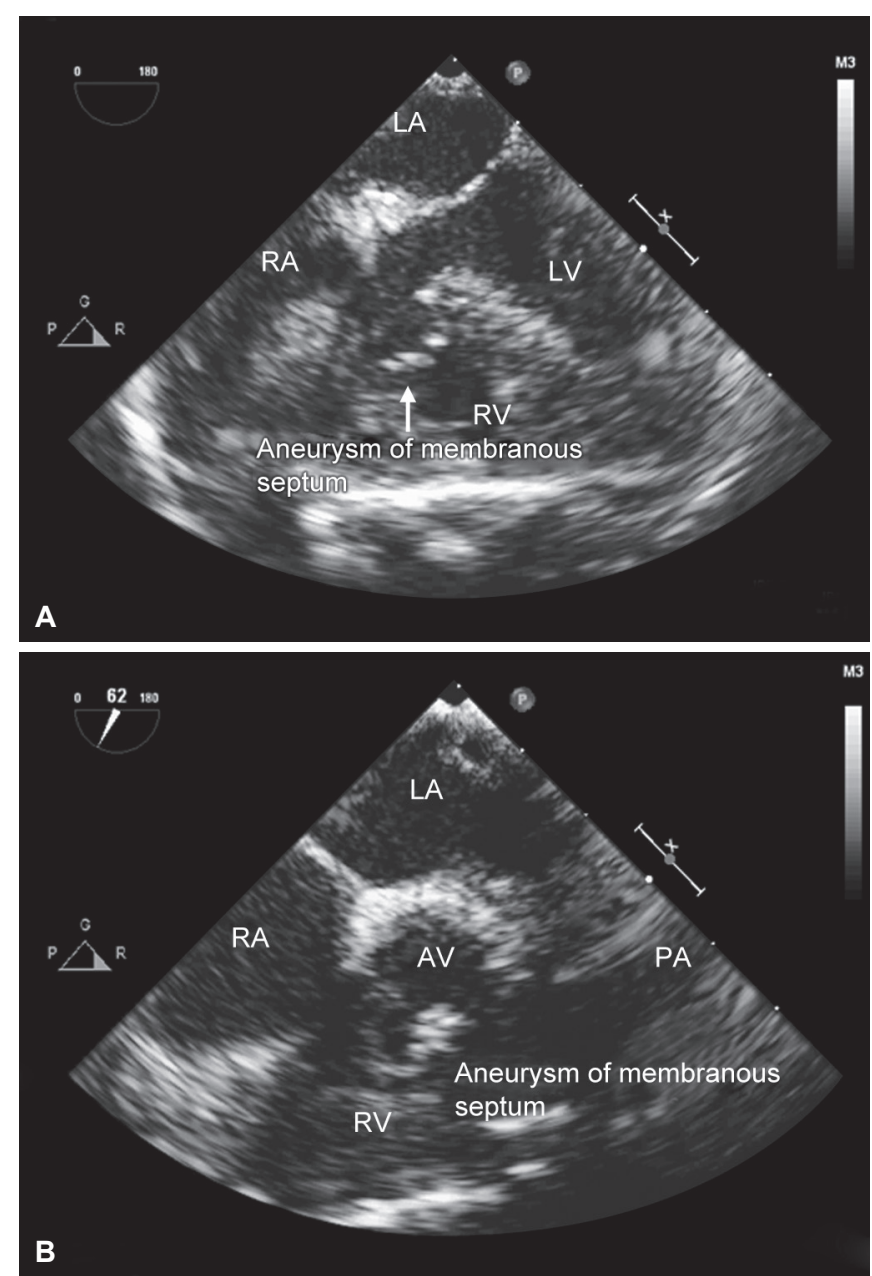

Figs 3A and B: (A) Midesophageal five-chamber view showing membranous septal aneurysm; (B) Midesophageal right ventricular inflow-outflow view showing membranous septal aneurysm

case, the aneurysm was confused with sinus of valsalva aneurysm, but it had no hemodynamic consequence. The aortic regurgitation was because of coaptation defect and not because of prolapse of cusp. The second case had valvular pulmonary stenosis, and aneurysm of 


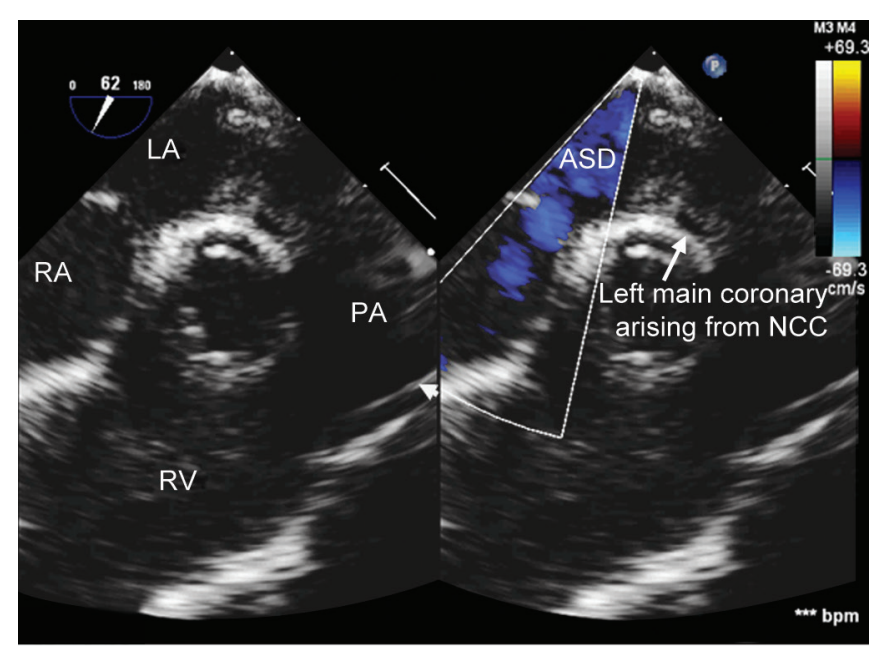

Fig. 4: Small atrial septal defect and left main coronary artery originating from noncoronary cusp

membranous ventricular septum was not causing any RVOT obstruction.

The membranous interventricular septum aneurysms can be diagnosed with cardiac catheterization. ${ }^{2}$ In the present age of echocardiography, cardiac catheterization is rarely necessary as a primary diagnostic modality for congenital heart disease. Echocardiography can diagnose these aneurysms, but it has a low sensitivity. ${ }^{3}$ Cardiac computed tomography and cardiac magnetic resonance imaging are other modalities that can be used for diagnosing the aneurysms of membranous interventricular septum. ${ }^{6,7}$

Transesophageal echocardiography is routinely done in patients undergoing cardiac surgery. Transesophageal echocardiography can accurately diagnose aneurysms of membranous ventricular septum. ${ }^{7,8}$ Various views should be used to confirm the diagnosis. Midesophageal five-chamber view usually shows the aneurysm. Midesophageal RV inflow-outflow view will show the aneurysm coming into RV cavity or RVOT during systole. Midesophageal long-axis view will show a communication between the left ventricle and the aneurysm and thus confirm the diagnosis. In all patients having an aneurysm of membranous interventricular septum, we should get Doppler of RVOT from transgastric view to rule out any RVOT obstruction. All aneurysms of the membranous interventricular septum should be excised so as to prevent complications. ${ }^{4}$ To conclude, aneurysms of membranous interventricular septum are rare, usually asymptomatic, and may occur with other cardiac lesions. They can be accurately diagnosed with TEE.

\section{REFERENCES}

1. Arons JJ, Orourke P. Myocardial infarction with aneurysm of interventricular septum and perforation. J Am Med Assoc 1954 Jul;155(12):1050-1052.

2. Edelstein J, Charms BL. Ventricular septal aneurysms. A report of two cases. Circulation 1965 Dec;32(6):1981-1984.

3. Canale JM, Sahn DJ, Valdes-Cruz LM, Allen HD, Goldberg SJ, Ovitt TW. Accuracy of two-dimensional (2D) echocardiography in the detection of aneurysms of the ventricular septum. Am Heart J 1981 Mar;101(3):255-259.

4. Yilmaz AT, Ozal E, Arslan M, Tatar H, Ozturk OY. Aneurysm of the membranous septum in adult patients with perimembranous ventricular septal defect. Eur J Cardiothorac Surg 1997 Feb;11(2):307-311.

5. Moe DG, Guntheroth WG. Spontaneous closure of uncomplicated ventricular septal defect. Am J Cardiol 1987 Sep; 60(8):674-678.

6. Naidu A, Ricketts M, Goela A, Shoemaker G, Li S. Incidental discovery of a membranous ventricular septal aneurysm in two dissimilar patients. Case Rep Cardiol 2012;2012: Article ID 324326:4 pages.

7. Fabijanic D, Bulat C, Batinic T, Carevic V, Caljkusic K. Membranous ventricular septum aneurysm as a cause of recurrent transient ischemic attack. J Cardiovasc Ultrasound 2012 Jun;20(2):114-115.

8. Sharma A, Kern MJ, Callicoat P, Aguirre F, Labovitz A, Willman VL. Severe subpulmonic outflow obstruction caused by aneurysm of the membranous ventricular septum: Diagnosis by transesophageal echocardiography. Am Heart J 1992 Mar;123(3):810-814. 